\title{
Diabetes Tipo 2 y Depresión en Guadalajara, México. 2005
}

\section{Type 2 diabetes and depression in Guadalajara, Mexico, 2005}

\author{
Cecilia Colunga-Rodríguez ${ }^{1}$, Javier E. García de Alba², \\ José G. Salazar-Estrada ${ }^{3}$ y Mario Ángel-González ${ }^{4}$
}

\footnotetext{
${ }^{1}$ Unidad de Investigación Médica, Hospital de Pediatría Centro Médico Nacional de Occidente. Instituto Mexicano del Seguro Social, Guadalajara, Jalisco México.

cecilia.colunga@imss.gob.mx, ccolungar@yahoo.com

2 Unidad de Investigación Social, Epidemiológica y de Servicios de Salud. Instituto Mexicano del Seguro Social. Guadalajara, México. jgarcia91046@yahoo.com

${ }^{3}$ Instituto de Salud Ocupacional. Departamento de Salud Pública. Universidad de Guadalajara, México.jsalazar@cucs.udg.mx

${ }^{4}$ Secretaría de Educación Pública, Estado de Jalisco, México. loupangel@yahoo.com.mx
}

Recibido 16 Julio 2007/Enviado para Modificación 7 Enero 2008/Aceptado 25 Enero 2008

\section{RESUMEN}

Objetivo Determinar la prevalencia de depresión y comparar indicadores sociodemográficos, metabólicos y clínicos, en personas con diabetes tipo 2 deprimidas y no deprimidas.

Material y métodos Se realizó un estudio transversal comparativo en una muestra de 450 personas con diabetes tipo 2, mayores de 30 años, con más de un año de diagnóstico y sin determinación de psicopatología. Aplicamos escala de Zung modificada y encuesta sociodemográfica, evaluamos Hemoglobina glucosilada, índice de masa corporal (IMC), tensión arterial y glucemia de ayuno; registramos antigüedad diagnóstica y estadio clínico de diabetes.

Resultados La prevalencia de depresión fue de $63 \%$, en una proporción de 3 a 1 , mayor en mujeres que en hombres con razón de momios 3,17(IC $95 \%$ 2,08-4,82) $p=0,0000$. Existen diferencias en escolaridad, estado civil y ocupación entre deprimidos y no deprimidos $(p<0.05)$, la edad no presenta diferencias, al igual que las variables metabólicas, excepto IMC, antigüedad diagnóstica y estadio clínico 3 y 4. En las variables socio-demográficas y clínico-metabólicas por sexo, no encontramos asociación en las primeras ( $>0.05)$, no obstante, antigüedad diagnostica y estadio clínico si se asocian con sexo, la primera de estas se asoció solo en hombres y la segunda en ambos sexos.

Conclusiones La prevalencia de depresión es alta en las personas con diabetes y las mujeres tienen mayor riesgo. La edad no muestra asociación entre personas con diabetes que están deprimidas, a diferencia de escolaridad, estado civil, y ocupación; la depresión se presentó más en personas con más antigüedad diagnóstica de diabetes y mayor IMC.

Palabras Clave: Depresión, diabetes, estudio comparativo (fuente: DeCS, BIREME). 


\begin{abstract}
Objective Determining the prevalence of depression and comparing sociodemographic, metabolic and clinical indicators in depressed and nondepressed people suffering from type 2 diabetes.

Methods A comparative cross-sectional study was carried out on a sample of 450 people aged over 30 suffering from type 2 diabetes; they had been diagnosed more than one year before and psychopathology had not been determined for them. A modified Zung's scale was applied and a sociodemographic survey carried out. Glucosylated haemoglobin, body mass index (BMI), blood pressure and glycemia in fasting were all evaluated. Diagnostic history and clinical state regarding diabetes were recorded.

Results There was $63 \%$ depression prevalence in a 3 to 1 ratio, this being greater in women than in men $(2,08-4,8295 \% \mathrm{Cl}, \mathrm{p}=0.00)$. Differences between depressed and non-depressed people were found in schooling, civil state and occupation $(p<0.05)$; differences were not presented with age, just like metabolic variables, except for IMC, diagnostic history and clinical states 3 and 4. No association was found between socio-demographic and clinical metabolic variables by gender ( $p>0.05$ ); however, diagnostic history and clinical state were associated with gender, the former being associated just with men and the latter with both sexes.

Conclusions The prevalence of depression was high in people suffering from diabetes and women were at greater risk. No association was revealed between age and people suffering from diabetes who were depressed, unlike schooling, civil state and occupation; depression was presented more amongst people having a longer diagnostic history of diabetes and greater BMI.
\end{abstract}

Key Words: Depression, diabetes, comparative study (source: DeCS, BIREME).

L

a diabetes mellitus tipo 2 (DM2) es un problema de salud pública a nivel global $(1,2)$ que ocasiona no solo daño a la salud física, sino que afecta la salud mental de quien la padece, se ha documentado una alta prevalencia de trastornos pico-afectivos, sobre todo depresión en enfermos crónicos (3-8). Lustman (9) refiere que la persona con diabetes puede desarrollar prácticamente cualquier síndrome psiquiátrico, siendo los trastornos más comunes la ansiedad y la depresión, no obstante, se conoce poco de la magnitud del problema de la depresión en pacientes de primer nivel de atención en cuanto a prevalencia y complejidad, ya que sólo $37 \%$ son detectados, quedando confusa la asociación con variables sociodemográficas y clínicometabólicas, principalmente hemoglobina glucosilada (HbA1c) e indicadores de control, además de patrones que guíen a etapas de evolución donde se presenta más frecuentemente la depresión, ya sea para prevenirla, o para disminuir o retrasar complicaciones. 
Los propósitos de este estudio fueron determinar la prevalencia de depresión y comparar indicadores sociodemográficos, metabólicos, antiguedad del diagnóstico de diabetes y estadio clínico en personas con diabetes tipo 2 con y sin depresión, usuarios de una Unidad de Medicina Familiar del Instituto Mexicano del Seguro Social en Guadalajara, Jalisco, México.

La depresión es el desorden afectivo más frecuente en población adulta y una de las más importantes causas de incapacidad en el mundo; la Organización Mundial de la Salud (OMS) considera que para el año 2020, según cita Borges, la depresión mayor unipolar, será la segunda causa de enfermedad más importante a nivel mundial (10). La depresión genera considerable sufrimiento a quienes la padecen, los problemas asociados a ella son extremadamente costosos a la sociedad (11) y una limitación para su tratamiento y control, es que frecuentemente pasa inadvertida (12). La prevalencia de depresión para población general, varía de 5 a $10 \%$, con una incidencia de casos nuevos de 13 $\%$ (13). Sin embargo, esta prevalencia entre los pacientes con diabetes es más elevada que en la población general (14), oscilando del $30 \%$ al $65 \%$ (3,7,12). Se ha demostrado que un $27 \%$ de personas con diabetes, pueden desarrollar depresión mayor en un lapso de 10 años, lo que sugiere que estas personas tienen más riesgo de sufrir trastornos psiquiátricos debido a factores relacionados con la diabetes, como el estrés de la cronicidad, la demanda de autocuidado y el tratamiento de complicaciones, entre otras (4).

Las personas con DM2 deprimidas, además de disminuir funcionalidad y calidad de vida, presentan problemas en el autocuidado y la interacción para atender su salud, por lo que sufrir depresión se asocia con pobre cumplimiento terapéutico, bajo control glucémico y riesgo incrementado para complicaciones micro y macro vasculares (15). En este sentido, se ha relacionado el control de la depresión con reducción de HbA1c de 0.8-1.2 \% (15,16), además se ha documentado un mayor riesgo de muerte por enfermedad coronaria en personas con diabetes que padecen depresión (11).

Por otra parte, el impacto de la diabetes en México, no solo se asocia con su magnitud epidémica, sino con las peculiaridades biológicas, psicológicas y sociales que caracterizan a los mexicanos con diabetes y las del sistema social y de atención a la salud en México (17). En la vivencia de la diabetes a partir del diagnóstico, algunos autores (18-21) mencionan un proceso psicológico hacia la aceptación de la enfermedad y la adherencia al tratamiento, que no necesariamente es igual en todas las personas. Entre las etapas del proceso se mencionan la negación, depresión, ira y negociación, 
por las que suele atravesar el paciente; sin embargo, en estas etapas, existen grandes agujeros negros en los que permanece por muchos años la persona o de los que nunca avanza para llegar a la aceptación, en la que hay más probabilidades de lograr autocontrol (22).

La tarea difícil del control metabólico condiciona aspectos socioculturales, psicológicos y educacionales, lo cual implica que lograr cifras glucémicas próximas a la normalidad, requiere de la participación activa del paciente donde el estado afectivo-emocional, puede ser la limitante o el apoyo para mantenerse motivado (23). Al respecto, algunos estudios sugieren la necesidad de contemplar aspectos psicosociales en la promoción de la salud y estrategias educativas, en grupos especialmente vulnerables como es el caso de los adultos con diabetes tipo 2 (24).

\section{MATERIAL Y MÉTODOS}

Se realizó un estudio transversal, comparativo en una muestra de pacientes derechohabientes del IMSS, adscritos a la Unidad de Medicina Familiar Número 3 de la ciudad de Guadalajara con diagnóstico de diabetes tipo 2, se identificó a las personas que presentaba sintomatología depresiva para compararlas con quienes no la tenían, de acuerdo a indicadores sociodemográficos, metabólicos y clínicos. El periodo de estudio comprendió de Enero de 2004 a Enero de 2005; los criterios de inclusión para los participantes fueron tener diagnóstico de DM2, realizado por el médico familiar, tener más de 30 años de edad a la fecha de diagnóstico y más de un año de antigüedad con la diabetes, expediente con exámenes clínicos completos y registros no mayores a 6 meses a la fecha de nuestro estudio; con los datos del expediente, clasificamos el estadio clínico de la diabetes, de acuerdo a la escala UISESS (25), que clasifica a los pacientes según el grado de evolución de la diabetes en: estadio 1, pacientes que sólo tienen diabetes de reciente diagnóstico y metabólicamente controlados; estadio 2 , pacientes con diabetes en descontrol metabólico; estadio 3, pacientes que tienen enfermedades agregadas a la diabetes, por ejemplo hipertensión; estadio 4, pacientes con complicaciones de la diabetes por ejemplo neuropatía, nefropatía y/o cardiopatía; estadio 5, pacientes con secuelas de las complicaciones de la diabetes, también en el expediente, verificamos que no tuvieran diagnóstico previo de depresión o de cualquier enfermedad psiquiátrica, ni estuvieran tomando tratamiento farmacológico antidepresivo.

Se estimó una muestra de 450 participantes, teniendo en cuenta la población total de pacientes con diagnóstico de diabetes, adscritos a la unidad médica de 
estudio y el número mínimo requerido para comparar dos grupos con niveles de significancia del $5 \%$. Se solicitó consentimiento informado y en los casos que se encontraron condiciones que requerían atención especializada, se informó al médico familiar para el envío del paciente al nivel y servicio correspondiente.

Para identificar sintomatología depresiva, se aplicó la Escala de Zung modificada por Calderón (26), este es un instrumento tipo Likert de 20 reactivos y cuatro opciones de respuesta que clasifica en niveles a la depresión, diferenciando a quienes no la tienen como normal, y en grados según su seriedad, como reacción de ansiedad, depresión moderada y severa, este instrumento concuerda con criterios diagnósticos para depresión de la OMS en su décima clasificación internacional de enfermedades (CIE-10), está adaptada transculturalmente y contiene los síntomas más importantes de depresión considerados en el DSM-IV como disforia, anhedonia, apatía y aislamiento (27), es una escala pequeña, autoaplicada y útil en estudios epidemiológicos. Para medir peso y talla utilizamos una báscula marca Torino persona Plus ${ }^{\circledR}$, con esta dímetro incluido, posteriormente, se calculó el índice de masa corporal (IMC), de acuerdo a la fórmula de peso en kilogramos entre talla en centímetros al cuadrado (peso kgs/talla $\mathrm{cms}^{2}$ ). La prueba de hemoglobina glucosilada (HbA1c), estándar de oro para control glucémico, se realizó con el analizador químico DCA-2000 de Bayer®. La tensión arterial (TA) se obtuvo con el baumanómetro automático marca Omron ${ }^{\circledR}$. Incluimos una cédula para registrar datos sociodemográficos y tiempo de diagnóstico de diabetes. Para estandarizar procedimientos, realizamos reuniones previas y durante el estudio, con el equipo que levantó los datos, capacitando a personal de medicina, nutrición y psicología en la aplicación de pruebas y revisión de expedientes clínicos.

El análisis de los datos se realizó con el programa estadístico para ciencias sociales (por sus siglas en inglés SPSS) versión 11.0, aplicando de acuerdo al tipo de variables, medidas de resumen, variabilidad y asociación así como estadísticos inferenciales: media, rango, desviación estándar; $\mathrm{Chi}^{2}$, análisis de varianza, comparación de proporciones y Razón de momios (OR) respectivamente, tomando como significativa una $\mathrm{p} \leq 0.05$.

\section{RESULTADOS}

Se estudiaron 450 personas con DM2, $62 \%$ pertenecientes al sexo femenino y $38 \%$ al masculino, con edad promedio de $60,4 \pm 10,7$ años, una escolaridad de $5 \pm 4$ años, estado civil $64 \%$ casados o con pareja y $36 \%$ viudos o sin pareja; la ocupación se distribuyó en 49 \% amas de casa, 19 \% pensionados, jubilados 
o desempleados y el resto (32 \%) con empleo remunerado. En variables metabólicas, encontramos el promedio de $\mathrm{HbA1c}$ de $8,4 \% \pm 2,1$, la glucosa de

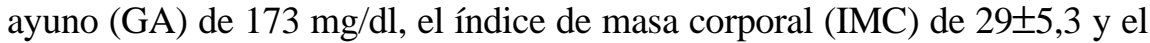
promedio de TA de $137 / 83 \pm 20 / 10 \mathrm{~mm} / \mathrm{Hg}$. El tiempo de diagnóstico de diabetes fue de 10,6 $\pm 7,6$ años y el estadio clínico de la enfermedad, de acuerdo a su evolución fue: personas en control de la diabetes $1 \%$, con descontrol metabólico $2 \%$, con enfermedades agregadas $28 \%$, con complicaciones de la diabetes 63 $\%$ y con secuelas de las complicaciones $6 \%$. Tanto HbA1c como GA, corresponden a lo que la Norma Oficial Mexicana (28), señala como descontrol, considerando controladas a quienes tienen $\mathrm{HbA1c} \leq 7 \%$ y glucemia de ayuno $\leq 126 \mathrm{mg} / \mathrm{dl}$, en tanto que la TA se puede considerar en control, tomando como parámetro cifras menor o igual a 140/90 mm/Hg, no así el IMC que en promedio corresponde a personas con algún grado de sobrepeso u obesidad.

Tabla 1. Sintomatología depresiva en la muestra

\begin{tabular}{lcc}
\hline \multicolumn{1}{c}{ Nivel } & No. & $\%$ \\
\hline Normal & 168 & 37 \\
Reacción de ansiedad & 115 & 26 \\
Depresión media & 137 & 30 \\
Depresión severa & 30 & 7 \\
\hline \multicolumn{1}{c}{ Total } & 450 & 100
\end{tabular}

Tabla 2. Diferencias entre pacientes diabéticos tipo 2 con o sin sintomatología

\begin{tabular}{|c|c|c|c|}
\hline Variable & $\begin{array}{l}\text { Con sintomatologia } \\
\text { depresiva } n=282\end{array}$ & $\begin{array}{l}\text { Sin sintomatologia } \\
\text { depresiva } n=168\end{array}$ & Observaciones \\
\hline Sexo \% & $\begin{array}{l}72 \text { femenino } \\
28 \text { masculino }\end{array}$ & $\begin{array}{l}45 \text { femenino } \\
55 \text { masculino }\end{array}$ & $\begin{array}{l}\text { OR }=3,17(\text { IC } 95 \% 2,08-4,82) \\
C h i^{2}=32,9 \\
P=0.000\end{array}$ \\
\hline Edad (promedio) & 60,9 años $\pm 10,7$ & 59,6 aก๊os $\pm 10,7$ & $\begin{array}{l}F=1,55 \\
P=0.21\end{array}$ \\
\hline $\begin{array}{l}\text { Escolaridad formal } \\
\text { (promedio) }\end{array}$ & 4,6 años $+4,4$ & 5,8 aก̃os $+4,4$ & $\begin{array}{l}F=7.74 \\
P=0.04\end{array}$ \\
\hline $\begin{array}{l}\text { Estado civil \% } \\
\text { - Casado o pareja } \\
\text { - Viudos sin pareja }\end{array}$ & $\begin{array}{l}61 \\
39\end{array}$ & $\begin{array}{l}70 \\
30\end{array}$ & $\begin{array}{l}\mathrm{Chi}^{2}=3,92 \\
\mathrm{P}=0.04\end{array}$ \\
\hline $\begin{array}{l}\text { Ocupación \% } \\
\text { - Con empleo } \\
\text { - Sin empleo }\end{array}$ & $\begin{array}{l}25 \\
75\end{array}$ & $\begin{array}{l}43 \\
57\end{array}$ & $\begin{array}{l}C h i^{2}=15,85 \\
P=0.000\end{array}$ \\
\hline
\end{tabular}

Fuente: Encuesta

Como se observa en la Tabla 1, sintomatología depresiva presenta una alta prevalencia en el total de la población (63 \% con algún nivel de depresión). Según se aprecia en la Tabla 2, el sexo y grupo de edad más afectados por la depresión son el femenino y los pacientes de 50 a 59 años, respectivamente. Aunque la edad no muestra diferencias significativas, la escolaridad, estado 
civil y ocupación, si muestran diferencias, señaladas por una $\mathrm{p} \leq 0.05$; en la distribución de la depresión por estadio clínico, encontramos uno de cada tres pacientes en estadios 3, 4 y 5 (25) correspondientes a enfermedad agregada, complicaciones y secuelas de las complicaciones, considerando que estas personas requieren de mayor atención psicológica, pensaríamos que un gran porcentaje necesita controlar su estado psicoafectivo por encontrarse con depresión severa. La mayoría de las variables clínico-metabólicas: HbA1c, GA, TA, no mostraron diferencias, sin embargo, IMC antigüedad diagnóstica y estadio clínico en niveles 3 y 4, mantuvieron diferencias estadísticas entre los deprimidos y los no deprimidos.

Como se indica en la Tabla 3, las variables socio-demográficas y clínicometabólicas analizadas por sexo, no sugieren asociación en las primeras $(\mathrm{P}>0,05)$, en tanto que antigüedad diagnostica y estadio clínico sí registraron asociación, en la primera de estas variables solo se encontró en los hombres y en la segunda en ambos sexos, también en los hombres, difiere el IMC menor a 30 con la presencia de depresión, en tanto que en las mujeres, existe diferencia tanto en el IMC mayor como en el menor de 30.

Tabla 3. Diferencias entre pacientes diabéticos tipo 2 con o sin sintomatología

\begin{tabular}{|c|c|c|c|}
\hline Variable & $\begin{array}{l}\text { Con sintomatologia } \\
\text { depresiva } n=282\end{array}$ & $\begin{array}{l}\text { Sin sintomatologia } \\
\text { depresiva } n=168\end{array}$ & Significancia \\
\hline Hemoglobina glucosilada $\%$ & $8,4 \pm 2,2$ & $8,4 \pm 2,1$ & $\begin{array}{l}F=0,04 \\
P=0.85\end{array}$ \\
\hline Glucemia de ayuno (mg/dl) & $174 \pm 77$ & $172 \pm 69$ & $\begin{array}{l}F=0,07 \\
P=0.78\end{array}$ \\
\hline Presión arterial $(\mathrm{mm} / \mathrm{Hg})$ & $137 / 82 \pm 20 / 10$ & $138 / 83 \pm 19 / 9$ & $\begin{array}{l}F=0,2 \\
P=0.6\end{array}$ \\
\hline $\begin{array}{l}\text { Índice de masa } \\
\text { corporal } \\
\text { (peso kgs/talla }{ }^{2} \text { ) }\end{array}$ & $29,3 \pm 5,4$ & $28,6 \pm 4,9$ & $\begin{array}{l}F=1,84 \\
P=0.17\end{array}$ \\
\hline $\begin{array}{l}\text { Antiguedad del diagnóstico } \\
\text { de DM2 (en años) }\end{array}$ & $11,5 \pm 8$ & $9,2 \pm 6,7$ & $\begin{array}{l}F=9,71 \\
P=0.001\end{array}$ \\
\hline Estadio clínico Diabetes \% & & & \\
\hline - Diabéticos en control & 1 & 1 & $P=0,73$ \\
\hline - Descontrol metabólico & 1 & 4 & $P=0.06$ \\
\hline - Enfermedad agregada & 25 & 34 & $\begin{array}{l}\mathrm{Chi}^{2}=4,31 \\
\mathrm{P}=0,03\end{array}$ \\
\hline - Complicaciones & 68 & 55 & $\begin{array}{l}\mathrm{Chi}^{2}=8,03 \\
\mathrm{P}=0.004\end{array}$ \\
\hline - Secuelas complicaciones & 5 & 6 & $\mathrm{P}=0.65$ \\
\hline
\end{tabular}




\section{DISCUSIÓN}

Encontramos similitudes con otras poblaciones estudiadas como la edad, predominio de mujeres y más de una década de antigüedad diagnóstica.

La TA en nuestro caso muestra cifras mejores a las reportadas en la Encuesta Nacional de Salud (>140/90), esto para la mitad de los casos (1), lo cual podría relacionarse con el consumo de medicamentos antihipertensivos para protección renal. En cuanto al IMC, corresponde a sobrepeso u obesidad (28), sin embargo, la fórmula para su medición no discrimina composición corporal, ya que el tejido magro es más pesado que el tejido adiposo (29), lo cual no logramos diferenciar; por otra parte, nuestros resultados en IMC sugieren diferencias entre hombres y mujeres $(p<0,001)$, siendo mayor en el sexo femenino el IMC y la depresión, la cual podría estar mediatizada por baja autoestima y por aspectos del género (30). Siguiendo con variables metabólicas, encontramos HbA1c de 8,5 \% correspondiente a descontrol, esto de acuerdo al Unit Kingdom Prospective Diabetes Study (UKPDS) (31), incrementa el riesgo de complicaciones micro y macro vasculares, por cada $1 \%$ de descontrol, no obstante, este descontrol es semejante al de países desarrollados como Estados Unidos y Reino Unido, lo que habla de las dificultades para el control de HbA1c en cifras óptimas ( $<7 \%$ ) a pesar de los avances tecnológicos y desarrollo económico de los países referidos.

Sin duda las modificaciones en los niveles de control metabólico se relacionan con el estilo de vida, cuyos dominios incluyen conductas y preferencias relacionadas con la alimentación, actividad física, consumo de alcohol, tabaco u otras drogas, responsabilidad para la salud, actividades recreativas, relaciones interpersonales, prácticas sexuales, actividades laborales y patrones de consumo (32), incluidas las formas de afrontamiento cognitivo y emocional ante situaciones estresantes como la enfermedad crónica, por lo que las modificaciones de estilo de vida para prevención y promoción de la salud, deben incluir el manejo emocional, ya que como lo menciona Franciosi, entre las conductas que el paciente debe adoptar para su autocontrol, el auto-monitorear el nivel glucémico, tiene relación significativa con niveles mayores de distrés, preocupaciones y síntomas depresivos (33), aunque Robert difiere al afirmar que a mayor sentido de autoeficacia ante la diabetes, disminuye el nivel de depresión y ansiedad, por lo que hay que empoderar a las personas para que sean autogestivas y se "apropien" de su enfermedad, responsabilizándose y controlando conductas de autocuidado (34), de acuerdo a lo anterior, la responsabilidad del tratamiento continuo de la diabetes recae directamente en el individuo y su familia, lo cual no resulta fácil para muchos pacientes, ya que las conductas de auto-gestión 
eficaces, son difíciles de lograr y mantener, muchas veces por el entorno social, las decisiones personales limitadas para cambiar estilo de vida y la confrontación inadecuada de problemas emocionales que acompañan el diagnóstico de la diabetes, las cuales son barreras a la terapia eficaz, asimismo, los sentimientos de enojo, culpa, resentimiento, miedo y tristeza, interfieren a menudo con la habilidad del individuo para el auto-cuidado exitoso.

En relación a la edad, existe una clara tendencia a pensar en que las personas después de los 50 años, tienden a deprimirse por razones pico-sociales variadas, algunas relacionadas con su estado de salud o por condiciones económicas o familiares adversas, sin embargo hay quienes mencionan que incluso los adolescentes se deprimen y encuentran la diabetes difícil de manejar (35), lo cual es afirmado por Kovacs al referir que existe mayor depresión y ansiedad generalizada en pacientes con diabetes (4), Anderson está de acuerdo con Kovacs al estimar que la depresión en personas con diabetes es el doble que en quien no la tiene y no difiere por circunstancias como sexo o tipo de diabetes (8), este último dato, se mantiene contrario a lo encontrado por nosotros, ya que sí identificamos diferencias por sexo, afectando más a las mujeres quienes presenta más riesgo de depresión en una proporción de 3 a 1 en relación a los hombres, lo anterior sugiere en nuestro contexto, un problema de género, esto lo apoya el estudio de Daniulaytité(36) quien realizó una investigación cualitativa en población del IMSS en Guadalajara y encontró que las mujeres con diabetes, tienen más dominio y mejor control respecto a su autocuidado que los hombre, lo que pudiera relacionarse a mayor depresión, además sus resultados sugieren que en prevención debe enfatizarse la integración de los hombres en el cuidado a la salud.

Se requiere más que un enfoque multidisciplinario, una tendencia a la transdisciplina, lo cual parece lejano en nuestras unidades de medicina familiar, debido a que no existen en estas clínicas, profesionales de la psicología, que lleven a la práctica estrategias de evaluación, intervención y seguimiento, en pacientes deprimidos en pro de una atención integral, y aunque en el Instituto Mexicano del Seguro Social, se reconoce la importancia de la atención psicológica en la diabetes, los psicólogos y psiquiatras, se ubican principalmente en segundo y tercer nivel atención y resultan insuficientes para atender a toda la población que requiere de estos servicios especializados. Asimismo, en servicios de salud, se deben desarrollar estrategias eficientes para atención de pacientes de mayor riesgo los cuales de acuerdo a este estudio, es 1 de cada 3, además de en casos necesarios, realizar el envío al nivel y servicio especializado para la prescripción de terapia farmacológica complementaria, ya que hay 
elementos para pensar que algunos pacientes deprimidos, pueden presentar algún desajuste bioquímico, así lo muestran hallazgos como los reportados por Vázquez, donde al parecer la ausencia de insulina modifica los procesos de síntesis de serotonina y dopamina cerebral (6).

Por otra parte, con fines preventivos, resulta conveniente seguir orientando los esfuerzos hacia investigaciones que busquen en los factores psicoafectivos elementos de riesgo para diabetes, ya que no ha quedado atrás la idea de que la depresión puede originar diabetes, así lo muestra el estudio de Carnethon (37) quien afirma que los síntomas depresivos pueden predecir un evento de diabetes, independientemente o a través de factores de riesgo establecidos para diabetes, un estudio que puede sustentar la anterior afirmación es el realizado en 2003 en Estados Unidos, donde se siguió una cohorte de 6190 hombres y mujeres de 25 a 74 años sin diabetes por 21 años, al término de los cuales, mostraron una incidencia mayor para diabetes quienes reportaron más sintomatología depresiva (38). De la misma forma, para un estudio más profundo del comportamiento de la depresión en la diabetes, se deben realizar estudios con diferentes metodologías, tal es el caso de estudios controlados, complementados con diseños cualitativos para conocer más de la subjetividad del padecimiento de quien vive con diabetes (22).

Podemos concluir que la prevalencia de depresión es de $63 \%$, más frecuente en mujeres que en hombres (3:1). El grupo de edad más afectado es de 50 a 59 años; sin embargo, la edad no muestra asociación entre las personas deprimidas a diferencia de la escolaridad, estado civil, y ocupación, la antigüedad diagnóstica sugiere diferencias en los deprimidos y los no deprimidos que al separar por sexo, mantiene diferencia para hombres, pero no para mujeres, no encontramos diferencias en depresión por sexo con HbA1c, glucemia de ayuno y TA, no obstante, el IMC si muestra diferencias entre hombres y mujeres deprimidos y quienes no lo están, estadio clínico presenta diferencias entre deprimidos y no deprimidos, esto solo en pacientes con enfermedad agregada y complicaciones que al separar por sexos, se comportaron con la misma diferencia *

Agradecimientos. Al Instituto Mexicano del Seguro Social por medio de la UMF 3, la Coordinación de Investigación en Salud y la Jefatura de prestaciones Médicas Delegación Jalisco, por su apoyo en la realización de este trabajo. 


\section{REFERENCIAS}

1.Velásquez MO, Rosas PM, Lara EA, Pastelín HG. Hipertensión arterial en México: Resultados de la Encuesta Nacional de Salud 2000 (ENSA 2000) Arch Cardiol Mex 2002; 72(1):71-84.

2. Aguilar CA, Velásquez O, Gómez F, GonzálezA, Lara A, Molina V, et al. Characteristics of patients with type 2 diabetes in Mexico. Diabetes Care 2003; (26):2021-2026.

3. Eaton WW, Armenian HA, Gallo J, Pratt L, Ford DE. Depression and risk for onset of type II diabetes: A prospective population-based study. Diabetes Care 1996; 19:1097-1102.

4. Kovacs M, Goldston D, Obrosky DS, Scott MS, Bonar LK. Psychiatric disorders in youths with IDDM: rates and risk factors. Diabetes Care 1997; 20(1):36-44.

5. Peyrot M, Rubin RR. Levels and risks of depression and anxiety symptomatology among diabetic adults. Diabetes Care 1997; 20:585-590.

6. Vázquez-Estupiñán F, Ruiz-Flores LG, Corlay-Noriega I. Depresión y diabetes mellitus: Conceptos actuales. Revista de Investigación en Salud 2000; 11:35-37.

7. Jacobson AM, Hauser ST, Willet JB, Dvorak R, Wolpert H, Herman L, et al. Pshychological adjustment to IDDM: 10-year follow-up of an onset cohort of child and adolescent patients. Diabetes Care 1997; 20(5):811-818.

8. Anderson RJ. The prevalence of comorbid depression in adults with diabetes: Ametaanalysis. Diabetes Care 2001; 24(6):1069-1078.

9. Lustman PJ, Griffith LS, Gavard JA, Clouse RE. Depression in adults with diabetes. Diabetes Care 1992; 15:1631-1639.

10. Borges G, Medina ME, López S. El papel de la epidemiología en la investigación de los trastornos mentales. Salud Pública de México 2004; 46:451-463.

11. Wagner FA, Gallo JJ, Delva J. Depression in late life: A hidden public health problem for México? Salud Pública de México 1999; 41(3):189-202.

12. López MI, Rodríguez HM. Propuesta de intervención para ancianos deprimidos. Revista Cubana de Medicina General Integr 1999; 15(1):19-23.

13. Santillana SP, Alvarado LE. Depresión en población adulta mayor: Tamizaje en unidad de primer nivel de atención médica. Rev Med IMSS 1999; 37(2):111-115.

14. Jouko A. Depression in subjects with type 2 diabetes: Predictive factors and relation to quality of life. Diabetes Care 1999; 22(6):997-998.

15. Lustman PJ. Depression and poor glycemic control: a meta-analytic review of the literature. Diabetes Care 2000; 23:934-942.

16. Lustman PJ. Fluoxetine for Depresión in Diabetes. A randomized double-blind placebocontrolled trial. Diabetes Care 2000; 23:618-623.

17. Alpizar SM, Pizaña BJA, Zárate AA. La diabetes mellitus en el adulto mayor. Rev Med IMSS 1999; 37(2):117-125. 
18. Instituto Nacional de Estadística Geografía e Informática (INEGI). XII Censo General de Población y Vivienda 2000. México, D.F. 2002.

19. Lerman GI. Aspectos psicosociales en la diabetes mellitus en: Atención integral del paciente diabético. México : Mc Graw Hill 2003 pp. 37-51.

20. Neel JV. The "thrifty genotype" in 1998. Nutrition Reviews 1999; 57(5):52-59.

21. Díaz Nieto L. Grupo de autocuidado de diabetes mellitus tipo II. Salud Pública de México 1993; 35(2):169-176.

22. Robles SL. ¿Cómo explicar el sufrimiento de las personas a causa de la enfermedad? Investigación en salud 1999; 3:205-212.

23. Lifshitz GA, Arreola F, Castro MG, Fiorelli S, Islas Andrade S, Méndez F. et al. A propósito de la diabetes en México. Rev Med IMSS 1996; 34(5):343-344.

24. Arauz AG. Intervención educativa comunitaria sobre la diabetes en el ámbito de la atención primaria. Rev Panam Salud Pública/Pan Am J Public Health 2001; 9(3):145-153.

25. García de Alba JE, Salcedo-Rocha AL, Colunga-Rodríguez C, González-Barrera JA, Herrera-Solís E, Milke-Nájar ME. UISESS Scale for Staging and Classifying clinical-Epidemiological risk in type 2 diabetes mellitus for establishing multidisciplinary preventive actions. Preventive Medicine 2005; 41:211-218.

26. Calderón-Narváez G. Cuestionario clínico para el diagnóstico de los cuadros depresivos. Rev Med IMSS 1992; 30:377-380.

27. American Psychiatric Association. Diagnostic and Statistical Manual of Mental Disorders 4th edition. Washington D.C. : American Psychiatric Association; 1994.

28. Secretaria de Salud Norma Oficial Mexicana. NOM-O15-SSA-2000. Para la prevención, tratamiento y control de la Diabetes Mellitus. México: Secretaría de Salubridad y Asistencia; 2000.

29. García de Alba JE, Salcedo-RochaAL, Covarrubias-Gutiérrez V, Colunga-Rodriguez C, Milke-Nájar ME. Diabetes mellitus tipo 2 y ejercicio físico: Resultados de una intervención. Rev Med IMSS 2004; 42(5):395-404.

30. Durán-Varela BR, Rivera-Chavira B, Franco-Gallegos E. Apego al tratamiento farmacológico en pacientes con diagnóstico de diabetes mellitus tipo 2. Salud Pública de México 2001; 43(3):233-236.

31. Unit Kingdom Prospective Diabetes Study Group (UKPDS). Intensive blood-glucose control with sulphonylureas or insulin compared with conventional treatment and risk of complications in patients with type 2 diabetes (UKPDSS 33). Lancet 1998;352:837-853.

32. López JM, Ariza CR, Rodríguez JR, Murguía C. Construcción y validación inicial de un instrumento para medir el estilo de vida en pacientes con diabetes mellitus tipo 2. Salud Pública Mex 2003; 45:259-268 
33. Franciosi M, Pellegrini F, De Berardis G, Belfiglio M, Cavaliere D, Di Nardo B, et.al. The Impact of Blood Glucose Self-Monitoring on Metabolic Control and Quality of Life in Type 2 Diabetic Patients. An urgent need for better educational strategies. Diabetes Care 2001; 24:1870-1877.

34. Robert M, Anderson MM, Funell MM, Fitzgerqald JT, Marrero DG. The diabetes empowerment scale: A measure of psychosocial self-efficacy. Diabetes Care 2000;23(6):739-743.

35. Grey M, Boland E, Yu C, Sullivan BS, Tamborlane W. Personal and family factors associated with quality of life in adolescents with diabetes. Diabetes Care 1998; 21:909-914.

36. Daniulaityte R. Making sense of diabetes: cultural models, gender and individual adjustment to type 2 diabetes in a Mexican community. Soc Sci Med 2004; 59:1899-1912.

37. Carnethon M, Kinder L, Fair J, Stafford R, Fortmann S. Síntomas de depresión como factor de riesgo para incidencia de diabetes: Hallazgos del estudio epidemiológico de seguimiento de salud Nacional y Examinación Nutricional, 1971-1992. American Journal of Epidemiology 2003; 158(5):416-423.

38. Black S, Markidez K, Ray L. Depresión predice el incremento en la incidencia de resultados adversos en personas de mayor edad Mexicoamericanos. Diabetes Care 2003; 26(10):2822-2828. 